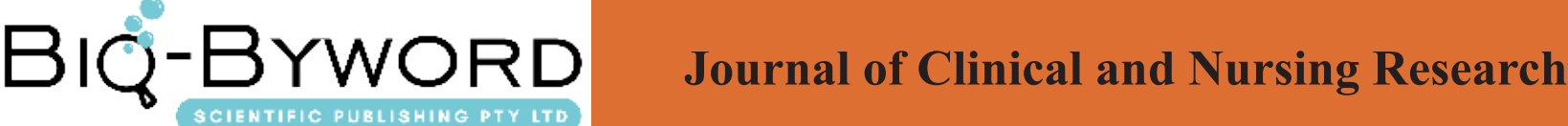

\section{Analysis of Nursing Intervention in Patients with Functional Gastrointes- tinal Diseases}

\author{
Song Jiaoyao \\ International Hospital, Peking University, Beijing 102206, China
}

\section{ARTICLE INFO}

Article history:

Published online: 15th July, 2017

Key words:

nursing intervention

functional gastrointestinal disease

\section{ABSTRACT}

Objective: To explore the effect of the nursing intervention in patients with functional gastrointestinal diseases. Methods: 79 functional gastrointestinal patients were selected from February 2015 to February 2016, and these patients were randomly divided into observation group and control group. 35 patients in each group. In the routine care of the medical department, the observation group has strengthened the comprehensive nursing care for the patient's psychological, the living diet, the living environment, and the patient's health education on the basis of the conventional nursing care. SCL-90 score was used to analyze the patient's care and observe the health of the two groups after nursing care. Results: The scores of SAS and SDS were compared between the two groups after the intervention of the two groups. The scores of the two groups were decreased before the nursing intervention, the difference was statistically significant, but the trend of the observation group was more obvious. Compared with the control group, compared with before treatment there is a certain difference, the difference was statistically significant. Conclusion: In the comprehensive care of gastrointestinal patients, it can obviously improve the anxiety and depression of functional patients and improve the quality of life of patients.

\section{Introduction}

Functional gastrointestinal disease is mainly caused by digestive system indigestion. Because the disease in the condition has a certain degree of intractability, and the recurrence of the disease more likely, so patients prone to a variety of adverse psychological emotions, easy to affect the clinical treatment of patients. According to the relevant clinical research shows that patients with functional dyspepsia comprehensive nursing management can effectively improve the patient in the treatment process of depression and other adverse psychological mood, improve the clinical treatment of patients with the effect. Now look at the following: 


\section{Materials and methods}

\subsection{General information}

We selected 70 functional gastrointestinal patients who were hospitalized from February 2015 to February 2016, and randomly divided these patients into observation group and control group. There were 16 males and 19 females in the observation group. The average age of these patients was 19-64 years, with an average age of $(40.36 \pm 4.67)$ years; 15 patients with functional dyspepsia, There are 13 patients with irritable bowel syndrome and 7 patients with functional constipation. Among the 35 patients in the control group, there were 17 males and 18 females, aged 19-63 years, with an average age of $(40.13 \pm 4.72)$ years and 16 patients with functional dyspepsia in the control group. Irritable bowel syndrome has 12, functional constipation in patients with 7 . There was no difference between the observation group and the control group in terms of endoscopy, biochemistry, and imaging examination, which met the relevant standard. Exclusion of gastrointestinal organic disease and a history of mental illness or disturbance of consciousness in patients.

\subsection{Methods}

After the two groups of patients after drug treatment, the control group using conventional care, observation group observation group in the care of patients, on the basis of routine care to strengthen the psychological, life diet, living environment, the patient Health education and other aspects of comprehensive care. Specific care methods are as follows:

(1) Diet care: in the diet of patients with care, in order to prevent the patient's digestive system to stimulate the situation, resulting in exacerbations in patients. Patients should develop good eating habits, in accordance with the provisions of the timetable to eat, reduce the patient due to the diet does not adapt to the situation.

(2) Psychological care: The hospital according to the existence of different social and psychological factors, analysis of the psychological situation of patients. According to the different circumstances of patients with care, so that patients in a comfortable, peaceful environment, so that patients in the ease of mind the condition can be effectively alleviated.

(3) Behavioral care: The main way for patients to self-minded to relax the psychological, so that patients with abdominal breathing way to effectively relieve abdominal muscles and allow patients to maintain a regular breathing movement, which can effectively regulate the patient's stomach function to help on patients' diet for early recovery of health.

(4) The effective treatment of patients with environmental care intervention: the patient's ward must ensure clean and sanitary. The patient's bed sheets need to keep dry and clean. The patient's ward must ensure a good lighting conditions and pay attention to the ward ventilation. Ward indoor air fresh, the patient living in the ward temperature control needs to be controlled at $18 \sim$ $25^{\circ} \mathrm{C}$, indoor humidity need to ensure that $50 \%$ to $60 \%$. So that patients in the treatment of the time to maintain a good mental state.

\subsection{Effect evaluation}

In the two groups of patients with nursing intervention, you can analyze the patient's anxiety and depression self-assessment score, to analyze the patient's anxiety and depression, SASS $\geq 50$ points for anxiety, SDS $\geq 50$ divided into depression, the higher the score, Indicating that patients with anxiety, depression, the more serious the situation.

\subsection{Statistical methods}

SPSS18.0 software was used for statistical analysis. Measurement data using $t$ test, $\mathrm{P}<0.05$ that the difference was statistically significant.

\section{Results}

Table 1 Comparison of SAS and SDS scores before and after intervention in both groups

\begin{tabular}{llllll}
\hline Group & $\mathrm{n}$ & \multicolumn{2}{c}{ SAS } & \multicolumn{2}{c}{ SDS } \\
\cline { 2 - 6 } & & $\begin{array}{l}\text { Before inter- } \\
\text { vention }\end{array}$ & $\begin{array}{l}\text { After interven- } \\
\text { tion }\end{array}$ & $\begin{array}{l}\text { Before inter- } \\
\text { vention }\end{array}$ & $\begin{array}{l}\text { After interven- } \\
\text { tion }\end{array}$ \\
\hline Control group & 35 & $50.78 \pm 11.47$ & $40.52 \pm 11.69^{*}$ & $52.37 \pm 11.78$ & $42.87 \pm 12.41^{*}$ \\
\hline Observation group & 35 & $51.09 \pm 11.68$ & $34.47 \pm 11.48^{*}$ & $53.14 \pm 11.42$ & $37.68 \pm 11.96^{*}$ \\
\hline
\end{tabular}


After the patients were treated, SAS and SDS scores were compared between the two groups. The scores of the two groups were decreased before the nursing intervention. The difference was statistically significant, but the trend of the observation group was more obvious than that of the control group, compared with before treatment there is a certain difference, the difference was statistically significant. See Table 1.

\section{Discussion}

Functional gastrointestinal indigestion. There are the following symptoms: abdominal distension, abdominal pain, early satiety, no appetite, nausea and so on. After clinical studies have found that functional gastrointestinal indigestion mainly with gastric acid secretion, Helicobacter pylori infection, abnormal gastric function caused by the situation. After this study can be found in the care of patients when the intervention, the use of comprehensive care can effectively alleviate the patient anxiety, depression and other emotions to help patients recover soon.

\section{Conclusion}

Thus, in the functional gastrointestinal dyspepsia patients care intervention, comprehensive care can effectively relieve the patient's negative emotions, which is conducive to the recovery of the patient's body and can get patient satisfaction, so the comprehensive nursing methods can be widely used clinically.

\section{References}

[1] Yang Yansheng, Peng Lihua. Functional Diagnostic Criteria and Research Status of Functional Gastrointestinal Diseases in Rome [J]. Journal of PLA Medical Sciences, 2013,34 (6): 541.

[2] Wang Jia Yu. Functional Dyspepsia 105 Cases of Treatment Experience $[\mathrm{J}]$. Chinese community physician, 2012,14 (31): 79-79.

[3] Li Qianling, Wu Juan. 72 Cases of Functional Gastrointestinal Care Nursing Experience [J]. Contemporary nurse special edition, 2008,20 (8): 15. 